\title{
Editorial
}

\section{La elastosonografía cuantitativa: una herramienta para evaluar el riesgo de malignidad del nódulo tiroideo}

\author{
Stella M. Batallés ${ }^{1}$ \\ 1 Jefe de Departamento de Diagnóstico por Imágenes del Instituto \\ Cardiovascular de Rosario, Argentina
}

Rev Argent Radiol 2021;85:81-82.

La elastografía cuantitativa (o "shear wave elastography" [SWE]) representa un importante avance en la evaluación ecográfica de la elasticidad del nódulo tiroideo.

Tal como expresan Uliaque Franco y col. ${ }^{1}$ en "Elastografía cuantitativa en la evaluación de nódulos tiroideos", publicado en el presente número, con esta técnica es posible medir la elasticidad de los tejidos independientemente de la presión aplicada por el operador, lo cual disminuye la variabilidad intra e interoperador respecto a la elastografía cualitativa.

La técnica utiliza ondas de corte inducidas por fuerza de radiación acústica centrada en un haz ultrasónico. La compresión provocada en el tejido es altamente localizada, ya que las ondas de corte inducidas son atenuadas por una región limitada cercana al centro del haz del ultrasonido. La onda final que recibe el transductor es la propagación de las ondas de corte, las cuales tienen relación con la dureza del tejido. Para medir la velocidad de las ondas de corte, el transductor debe mensurar la respuesta de al menos dos puntos $\mathrm{y}$, mediante algoritmos, obtener la velocidad, teniendo el desplazamiento y el tiempo. Al reconstruir la onda de propagación de velocidad del tejido en la región desplazada y utilizando algoritmos de correlación cruzada, se obtienen imágenes en tiempo real, en escala de colores y la elasticidad de la región de interés en unidades de presión (Kilopascales [kPa]) o en unidades de velocidad (m/seg, cm/seg). ${ }^{2}$

Numerosos trabajos científicos han avalado la utilidad de la técnica para diferenciar los nódulos más duros de los más elásticos, considerando que la rigidez aumenta la sospecha de malignidad de los nódulos en cuestión. ${ }^{3-6}$

En el análisis de la bibliografía se hallan rangos variables de sensibilidad y especificidad del método que oscilan entre 57 y $86 \%$ y entre 85 y 93,9\%, respectivamente (-Tabla 1). ${ }^{7}$
Uliaque Franco y col. ${ }^{1}$ expresan en su investigación que gran parte de la literatura sugiere que la elastografía muestra su mayor utilidad en el diagnóstico de nódulos benignos y hacen referencia a trabajos como los de Kagoya y col, ${ }^{8}$ Luo y col. $^{4}$ y Nell y col, ${ }^{9}$ que llegan a esa conclusión. Si consideramos la fortaleza de la técnica en detectar los verdaderos nódulos benignos, estaría cumpliendo con la premisa de que la elastografía sirve para evitar biopsias innecesarias. Ahora bien, corresponde analizar qué conducta tomamos si nos encontramos frente a un nódulo con variables ecográficas sugestivas de malignidad (microcalcificaciones, nódulo más alto que ancho, contornos imprecisos etc.) que se muestra elástico o con bajos valores de presión. De ninguna manera dejamos de indicar la PAAF. Entonces, cabe recordar que ninguna variable ecográfica por sí misma tiene una precisión diagnóstica de cáncer superior al 75\% (y el comportamiento elastográfico del nódulo no escapa a esta premisa). ${ }^{10}$ Por ello, actualmente, se considera la elastosonografía como una herramienta más en el análisis ultrasonográfico del nódulo tiroideo.

Sabemos que existen varios sistemas de clasificación de riesgo ecográfico del nódulo tiroideo (TIRADS), de los cuales los principales son el propuesto por el Colegio Americano de Tiroides (ACR), la Asociación Americana de Tiroides (ATA), el Sistema de Clasificación Chileno, European (EU) TI-RADS, Korean (K) TI-RADS, clasificación de la American Association of Clinical Endocrinologists (AACE), American College of Endocrinology (ACE), Associaziones Medici Endocrinologi (AME) y la British Thyroid Association (BTA). ${ }^{1-17}$ La pregunta es por qué ninguno de ellos ha incluido la elastosonografía como variable indicativa de PAAF. Quizás en las nuevas versiones - y a la luz de nuevas investigaciones - pueda ocupar su lugar en dichos scores.

\author{
Address for correspondence \\ Stella M. Batallés, Jefe de \\ Departamento de Diagnóstico \\ por Imágenes del Instituto \\ Cardiovascular de Rosario, \\ Argentina, Titular de la Cátedra \\ de Diagnóstico por Imágenes de \\ la Universidad Abierta \\ Interamericana (UAI), Rosario, \\ Argentina \\ (e-mail: batalless@gmail.com).
}

DOI https://doi.org/ 10.1055/s-0041-1740535. ISSN 1852-9992.

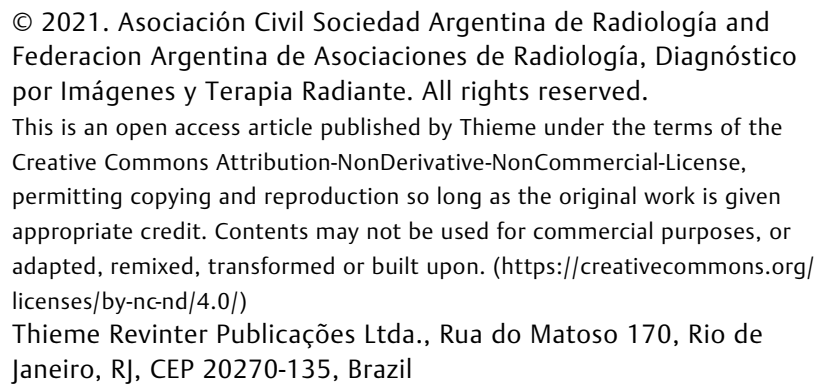


Tabla 1 Capacidad de la elastosonografía cuantitativa (SWE) para diferenciar nódulos benignos y malignos (Modificado de 7)

\begin{tabular}{|l|l|l|}
\hline & Sensibilidad & Especificidad \\
\hline Sebag F y col. (2010) & $85,2 \%$ & $93,9 \%$ \\
\hline Gu J y col. (2012) & $86 \%$ & $93 \%$ \\
\hline Veyrieres J y col. (2012) & $57 \%$ & $85 \%$ \\
\hline Zhang B y col. (2013) & $84 \%$ & $90 \%$ \\
\hline Kim H y col. (2013) & $80 \%$ & $90,5 \%$ \\
\hline Liu B y col. (2014) & $68,4 \%$ & $86,7 \%$ \\
\hline Lin P y col. (2014) & $84,3 \%$ & $88,4 \%$ \\
\hline
\end{tabular}

Continuando con los sistemas de clasificación de riesgo ecográfico, en el diseño del trabajo publicado en este número, hubiese sido interesante investigar la relación entre los valores de presión de los nódulos y el score de TIRADS al cual pertenecen, similar a lo investigado por Paredes Manjarrez y col, ${ }^{18}$ quienes hallaron que nódulos TI-RADS 1-4 con valores de rigidez por SWE menores a 21,6 kPa tuvieron mayor probabilidad de ser benignos, mientras que los nódulos tiroideos TI-RADS 5 con rigidez $>32,5 \mathrm{kPa}$ mostraron mayor riesgo de ser malignos. Otras investigaciones demostraron que el análisis conjunto del TIRADS, más los valores de presión en la SWE, puede tener una especificidad de hasta $95,8 \%$ y una sensibilidad de $76,29 \%$ en comparación con TIRADS solo y la SWE sola, cuyos valores de sensibilidad y especificidad son menores. ${ }^{19}$

Varios autores han reportado el valor de la elastografía, especialmente en aquellos nódulos con citología indeterminada en la PAAF. ${ }^{20,21}$ Creo que ese sería el foco para concentrarse en las investigaciones, analizando si realmente la elastosonografía puede influir para decidir la conducta terapéutica posterior en un nódulo clasificado como Bethesda III y IV en la citología, aunque ya es sabida la baja capacidad de la técnica para diferenciar adenoma de carcinoma folicular. ${ }^{22}$

Por otro lado, destaco la importancia de la toma de biopsia guiada por elastografía, ya que ha demostrado ser de ayuda para disminuir los resultados falsos negativos de la PAAF. ${ }^{18}$

\section{Bibliografía}

1 Uliaque Franco C, Herrero Laborda R, Hervias Ángulo E, Almenara Riaguas A, Berdún Pardo FJ. Elastografía cuantitativa en la evaluación de nódulos tiroideos. Rev Argent Radiol 2021;85(04):XX-XX

2 Castaneda B, Ormachea J, Rodríguez P, Parker KJ. Application of numerical methods to elasticity imaging. Mol Cell Biomech 2013; 10(01):43-65

3 Kura M, Ballarino C, Tamagnone F, Campagno B, Bertini K, Gómez $\mathrm{J}$, et al. Relación entre el valor del ratio elastográfico y la clasificación citológica de Bethesda en la patología tiroidea. Rev Argent Radiol 2014;78(03):128-137

4 Luo S, Kim EH, Dighe M, Kim Y. Thyroid nodule classification using ultrasound elastography via linear discriminant analysis. Ultrasonics 2011;51(04):425-431

5 Zhao CK, Xu HX. Ultrasound elastography of the thyroid: principles and current status. Ultrasonography 2019;38(02):106-124

6 Veyrieres JB, Albarel F, Lombard JV, Berbis J, Sebag F, Oliver C, et al. A threshold value in Shear Wave elastography to rule out malignant thyroid nodules: a reality? Eur J Radiol 2012;81(12):3965-3972
7 Blando A. Ecografía de cuello: Tiroides, Paratiroides, Salivales, Ganglios linfáticos y Otras neoplasias. $2^{\circ}$ Edición. Buenos Aires: Ediciones Journal; 2017:90

8 Kagoya $\mathrm{R}$, Monobe $\mathrm{H}$, Tojima $\mathrm{H}$. Utility of elastography for differential diagnosis of benign and malignant thyroid nodules. Otolaryngol Head Neck Surg 2010;143(02):230-234

9 Nell S, Kist JW, Debray TP, de Keizer B, van Oostenbrugge TJ, Borel Rinkes $\mathrm{IH}$, et al. Qualitative elastography can replace thyroid nodule fine-needle aspiration in patients with soft thyroid nodules. A systematic review and meta-analysis. Eur J Radiol 2015;84(04):652-661

10 Moon WJ, Jung SL, Lee JH, Na DG, Baek JH, Lee YH, et al;Thyroid Study Group, Korean Society of Neuro- and Head and Neck Radiology. Benign and malignant thyroid nodules: US differentiation-multicenter retrospective study. Radiology 2008;247(03):762-770

11 ACR TI-RADS Steering Committee. American College of Radiology Web Site. https://www.acr.org/Clinical-Resources/Reportingand-Data-Systems/TI-RADS Accessed October 20, 2021

12 Haugen BR, Alexander EK, Bible KC, Doherty GM, Mandel SJ, Nikiforov YE, et al. 2015 American Thyroid Association Management Guidelines for Adult Patients with Thyroid Nodules and Differentiated Thyroid Cancer: The American Thyroid Association Guidelines Task Force on Thyroid Nodules and Differentiated Thyroid Cancer. Thyroid 2016;26 (01):1-133

13 Horvath E, Majlis S, Rossi R, Franco C, Niedmann JP, Castro A, Dominguez M. An ultrasonogram reporting system for thyroid nodules stratifying cancer risk for clinical management. J Clin Endocrinol Metab 2009;94(05):1748-1751

14 Russ G, Bigorgne C, Royer B, Rouxel A, Bienvenu-Perrard M. The thyroid imaging reporting and data system (TIRADS) for ultrasound of the thyroid. J Radiol 2011;92(7-8):701-713

15 Shin JH, Baek JH, Chung J, Ha EJ, Kim JH, Lee YH, et al. Korean Society of Thyroid Radiology (KSThR) and Korean Society of Radiology. Ultrasonography Diagnosis and Imaging-Based Management of Thyroid Nodules: Revised Korean Society of Thyroid Radiology Consensus Statement and Recommendations. Korean J Radiol 2016;17(03):370-395

16 Gharib H, Papini E, Valcavi R, Baskin HJ, Crescenzi A, Dottorini ME, et al;AACE/AME Task Force on Thyroid Nodules. American Association of Clinical Endocrinologists and Associazione Medici Endocrinologi medical guidelines for clinical practice for the diagnosis and management of thyroid nodules. Endocr Pract 2006;12(01):63-102

17 Perros P, Boelaert K, Colley S, Evans C, Evans RM, Gerrard Ba G, et al. British Thyroid Association. Guidelines for the management of thyroid cancer. Clin Endocrinol (Oxf) 2014;81(Suppl 1):1-122

18 Paredes-Manjarrez C, Arreola-Cháidez D, Magdalena-Buitrago A, Ferreira-Hermosillo A, Avelar-Garnica JF, Arreola-Rosales R. Elastografía por ondas de corte como herramienta en la evaluación de los nódulos tiroideos. Gac Med Mex 2021; 157:19-24

19 Cantisani V, David E, Grazhdani H, Rubini A, Radzina M, Dietrich $\mathrm{CF}$, et al. Prospective evaluation of semiquantitative strain ratio and quantitative 2D ultrasound shear wave elastography (SWE) in association with TIRADS classification for thyroid nodule characterization. Ultraschall Med 2019;40(04):495-503

20 Rago T, Vitti P. Role of thyroid ultrasound in the diagnostic evaluation of thyroid nodules. Best Pract Res Clin Endocrinol Metab 2008;22(06):913-928

21 Mehrotra P, McQueen A, Kolla S, Johnson SJ, Richardson DL. Does elastography reduce the need for thyroid FNAs? Clin Endocrinol (Oxf) 2013;78(06):942-949

22 Bhatia KS, Lee YY, Yuen EH, Ahuja AT. Ultrasound elastography in the head and neck. Part II. Accuracy for malignancy. Cancer Imaging 2013;13(02):260-276 